\title{
Analysis of Strain Relaxation and Emission Spectrum of A Free-Standing GaN-based Nanopillar
}

\author{
Yuh-Renn $\mathrm{Wu}^{a}$, Peichen $\mathrm{Yu}^{b}$, C.H. Chiu ${ }^{b}$, Cheng-Yu Chang ${ }^{a}$, and H.C. Kuo ${ }^{b}$ \\ ${ }^{a}$ Institute of Photonics and Optoelectronics and Department of Electrical Engineering, \\ National Taiwan University, Taipei, Taiwan, 10617. \\ ${ }^{b}$ Department of Photonics and Institute of Electro-Optical Engineering, National Chiao-Tung \\ University, Hsinchu, Taiwan, R.O.C.
}

\begin{abstract}
We have made a GaN-based single nanopillar with a diameter of 300nm using the focused ion beam (FIB) technique. The micro-photoluminescence $(\mu$-PL) from the embedded GaN/InGaN multi-quantum wells reveals a blue shift of $68.3 \mathrm{meV}$ in energy. In order to explain the spectrum shift, we have developed a valence force field model to study the strain relaxation mechanism in a single GaN-based nanopillar structure. The strain distribution and strain induced polarization effect inside the multiple quantum wells is added to our self-consistent Poisson, drift-diffusion, and Schrodinger solver to study the spectrum shift of $\mu$-PL.
\end{abstract}

Keywords: GaN, InGaN, nanopillar, nanorod, strain relaxation, valence force field model

\section{INTRODUTION}

The III-nitrides have become very important materials for applications in optoelectronic ${ }^{1-5}$ and high power electronics. ${ }^{6,7}$ The III-nitrides are direct bandgap materials which can directly emit light easily. The bandgap of nitride alloys is from $6.2 \mathrm{eV}(\mathrm{AlN})$ to $0.7 \mathrm{eV}(\mathrm{InN})$ which covers from the UV light to the infrared. These properties make it promising for many applications. The III-nitrides have now been widely applied in the quantum well based light emitting diodes(LED) for UV and blue light sources. With the assistant of phosphors, it is possible to convert the UV or blue light into green and red light so that making a white light source for LED becomes feasible. However, it is difficult to directly make a green or a red quantum well LEDs with InGaN based materials. As we know, there is a large lattice mismatch $\sim 10 \%$ between $\mathrm{InN}$ and GaN. This large lattice mismatch leads to a strong piezoelectric polarization field inside the quantum wells for the normally used c-axis growth. As a result, the strong quantum confined Stark effect (QCSE) is observed in the quantum well structures. The crystal quality also worsens due to stronger strain as we push the emission to longer wavelengths by increasing the indium composition.

The strain related issues are difficult to be avoided since it always appears in during the epitaxial growth of InGaN with an large area size. However, the development of nanotechnology has enabled the study of the optical properties of the nanostructures. GaN-based nanostructures, such as nanorods, nanocones, or nanopillars, have been fabricated via various methods. ${ }^{8-12}$ Several characterizations have been made on the properties of these structures. However, few studies have clarified the emission mechanism from one single nanopillar. In our recent work, ${ }^{13}$ we have made a GaN-based single nanopillar with a diameter of $300 \mathrm{~nm}$ using the focused ion beam (FIB) technique. The micro-photoluminescence ( $\mu$-PL) from the embedded GaN/InGaN multi-quantum wells (MQWs) reveals a blue shift of $68.3 \mathrm{meV}$ in energy. The full width at half maximal (FWHM) is also broadened due to the distribution of strain relaxation in the nanopillar.

In this paper, we have developed a valence force field model to to explain the $\mu$-PL spectrum in detail and study the strain relaxation mechanism in a single GaN-based nanopillar structure. The strain distribution and strain induced polarization effect inside the multiple quantum wells is added to our self-consistent Poisson, drift-diffusion, and Schrodinger solver to study the spectrum shift and spectrum broadening effect of $\mu$-PL. The emission spectrum under different strain and different carrier injection condition will be calculated and fitted into the experimental results.

Further author information: (Send correspondence to Yuh-Renn $\mathrm{Wu}$ ) Yuh-Renn Wu: E-mail: yrwu@cc.ee.ntu.edu.tw, Telephone: 886-2-33669647

Eighth International Conference on Solid State Lighting,

edited by lan T. Ferguson, Tsunemasa Taguchi, lan E. Ashdown, Seong-Ju Park,

Proc. of SPIE Vol. 7058, 70580G, (2008) · 0277-786X/08/\$18 · doi: 10.1117/12.800658

Proc. of SPIE Vol. 7058 70580G-1 


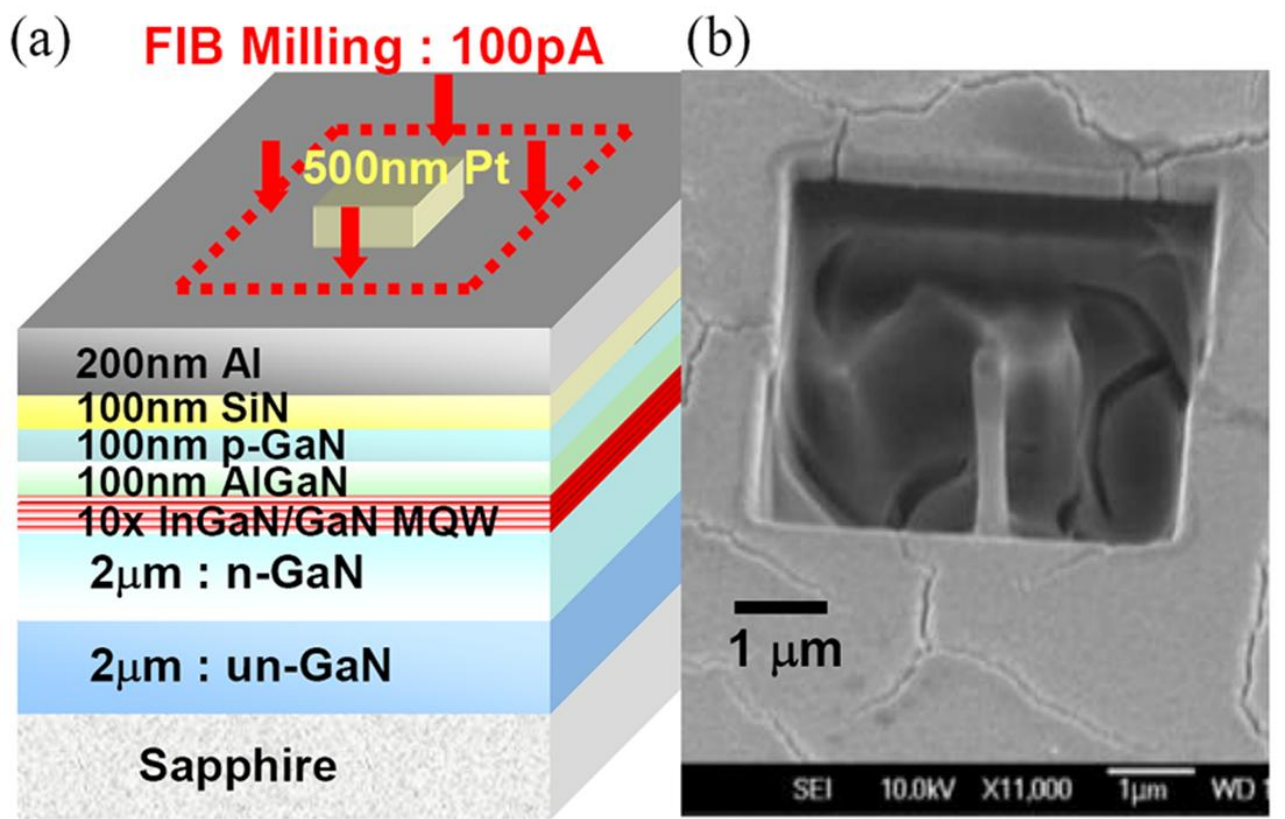

Figure 1. (a) Schematic of the epitaxial structure and the deposited materials for nanopillar fabrication using a focused ion beam. (b) The field-emission scanning-electron micrographic (FESEM) image of the fabricated free-standing nanopillar with a diameter of $300 \mathrm{~nm}$ and a length of $2 \mu \mathrm{m}$.

\section{EXPERIMENTS}

In our previous work, ${ }^{13}$ we have made a nano-pillar structures as shown in Fig. 1(a). The epitaxial structure of the device consists of a $2-\mu \mathrm{m}$-thick GaN nucleation layer, a $2-\mu \mathrm{m}$-thick Si doped n-GaN cladding layer, ten periods of $\operatorname{In}_{0.2} \mathrm{Ga}_{0.8} \mathrm{~N}$ MQWs of $2.5 \mathrm{~nm}$ thickness separated by a GaN barrier of 10nm thickness, a 100-nm-thick AlGaN cladding layer, and a 100-nm-thick Mg-doped p-GaN layer. To prepare for the FIB milling, a 100-nmthick $\mathrm{SiN}_{x}$ was deposited by plasma enhanced chemical vapor deposition (PECVD), followed by a 200-nm-thick $\mathrm{Al}$ layer using electron-beam evaporation. Then a platinum $(\mathrm{Pt})$ mask with a thickness of $500 \mathrm{~nm}$ was formed by the FIB deposition mode in an area of $500 \mathrm{~nm} \times 500 \mathrm{~nm}$ on the Al layer to provide localized protection from the ion beam sputtering. Finally, a $30-\mathrm{keV}$ Ga ion beam with a current of $100 \mathrm{pA}$ was applied to remove the material around the Pt mask in a four-step milling process. Fig. 1(b) shows the field-emission scanning-electron micrograph (FESEM) of the fabricated single nanopillar. The fabricated well area was approximately $5 \times 5 \mu \mathrm{m}^{2}$, while the dimensions of the fabricated nanopillar were $300 \mathrm{~nm}$ in diameter and $\sim 2 \mu \mathrm{m}$ in height. The $\mu$-PL characteristics were investigated for the fabricated device with and without the Pt mask at room temperature. The device was first put on a piezoelectric translator stage with a resolution of $2 \mathrm{~nm}$, where the He-Cd laser beam with a wavelength of $325 \mathrm{~nm}$ was focused on to a spot size of $\sim 3 \mu \mathrm{m}$ by a $40 \times$ objective lens with a numerical aperture (NA) of 0.5. The emission was then collected by the same objective lens, transmitted to a charge-coupled device (CCD) and a $0.32 \mathrm{~m}$ spectrometer, and measured at a spectral resolution of $0.1 \mathrm{~nm}$. After collecting the $\mu$-PL spectrum and the scanning image of the nanopillar with the Pt mask, the mask was removed by the metal etcher and the same structure was scanned again. The emission from the nano-pillar was collected by the same objective lens, and transmitted to a $0.32 \mathrm{~m}$ spectrometer with a spectral resolution of $0.1 \mathrm{~nm}$ and a charge-coupled device (CCD). The emission image is shown in Fig. 2.

\section{THEORETICAL MODEL}

In order to study the strain inside the nano-pillar, we apply our previously developed simulation program based on a valence force field(VFF) model. ${ }^{14}$ The VFF model is a microscopic model where the interaction between each atom and its nearby atoms is considered. The total strain energy is expressed as function of atomic positions, 
(a)

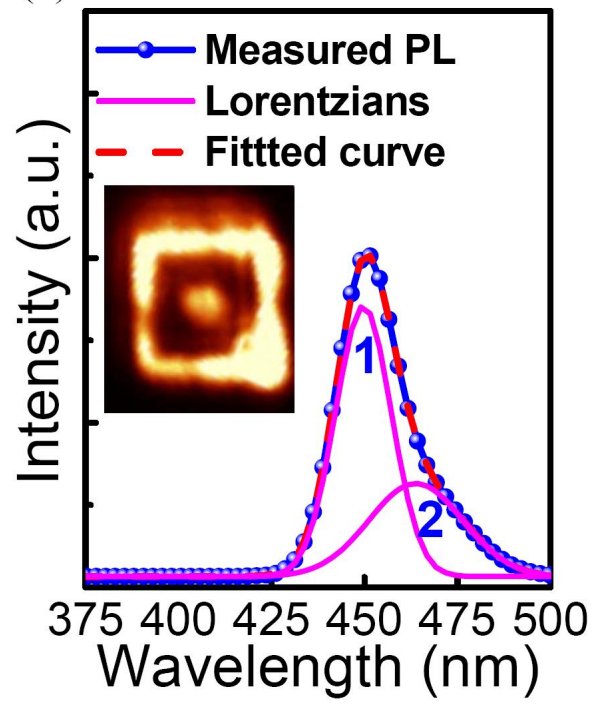

(b)

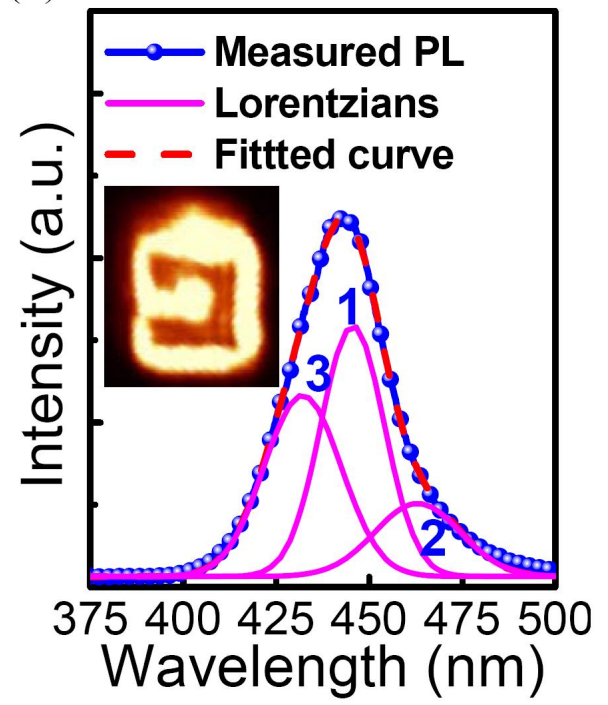

Figure 2. (a) The measured micro-photoluminescence ( $\mu$-PL) spectrum from the free-standing nanopillar with the Pt mask and (b) without the Pt mask. The insets show the respective scanning CCD images of the device. Lorentzian analyses of the measured spectra reveals additional photoluminescence, denoted as Lorentzian 3, in the shorter wavelength region, which originates from the free-standing nanopillar.

$\vec{R}_{i}$, using a sum of bond stretching $\left(V_{2}\right)$ and bond bending $\left(V_{3}\right)$ terms:

$$
\begin{aligned}
E_{A E}= & \sum_{i j} V_{2}\left(\vec{R}_{i}-\vec{R}_{j}\right)+\sum_{i j k} V_{3}\left(\hat{\theta}_{i j k}\right) \\
= & \frac{1}{2} \sum_{i} \sum_{j}^{n n} \frac{3 \alpha_{i j k}}{8\left(d_{i j}^{0}\right)^{2}}\left[\left(\vec{R}_{i}-\vec{R}_{j}\right)^{2}-\left(d_{i j}^{0}\right)^{2}\right]^{2} \\
& +\frac{1}{2} \sum_{i} \sum_{j, k>j}^{n n} \frac{3 \beta_{i j k}}{8 d_{i j}^{0} d_{i j}^{0}}\left[\left(\vec{R}_{j}-\vec{R}_{i}\right) \cdot\left(\vec{R}_{k}-\vec{R}_{i}\right)-\cos \theta_{0} d_{i j}^{0} d_{i j}^{0}\right]^{2},
\end{aligned}
$$

where $d_{i j}^{0}$ denotes the unstrained bond length between atoms $\mathrm{i}$ and $\mathrm{j}$, and $\theta_{0}$ is the unstrained bond angle, and $\cos \theta_{0}=-1 / 3$. The bond stretching $\alpha$ and bond bending $\beta$ force constants are listed in Mattila et. al. ${ }^{14}$ The strain energy of each atom is minimized by an iterative method to find the most stable configuration. Once the system reaches the lowest energy, the strain distribution inside the structure can be obtained.

After obtained the strain distribution of the quantum well inside the nano-pillar structure, we can obtain the piezoelectric polarization induced by strain by

$$
P_{e z}(x, y, z)=e_{31}\left(\epsilon_{x x}(x, y, z)+\epsilon_{y y}(x, y, z)\right)+e_{33} \epsilon_{z z}(x, y, z),
$$

where $e_{31}$ and $e_{33}$ are the piezoelectric coefficient and can be found in Ambacher, et. al. ${ }^{15}$ After obtaining the piezoelectric polarization, the fixed polar charge can be obtained from the difference of piezoelectric and spontaneous polarization field.

Since the $P_{e z}$ is a distribution function of $\mathrm{x}, \mathrm{y}$, and $\mathrm{z}$, we can calculate the piezoelectric polarization induced charges of each unit cell by

$$
\rho_{p z}(x, y, z)=\left(P_{e z}(x, y, z+d z)-P_{e z}(x, y, z)\right) / d z .
$$

The calculated distribution of the polar charges inside the multiple quantum well is then used in the self-consistent Poisson, drift-diffusion, and Schrodinger solver developed in our lab ${ }^{16}$ to study the band diagram, the emission 


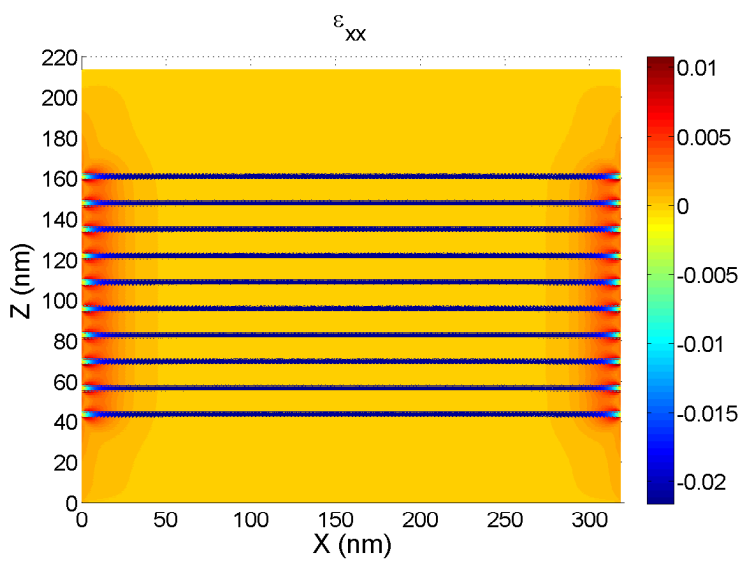

(a)

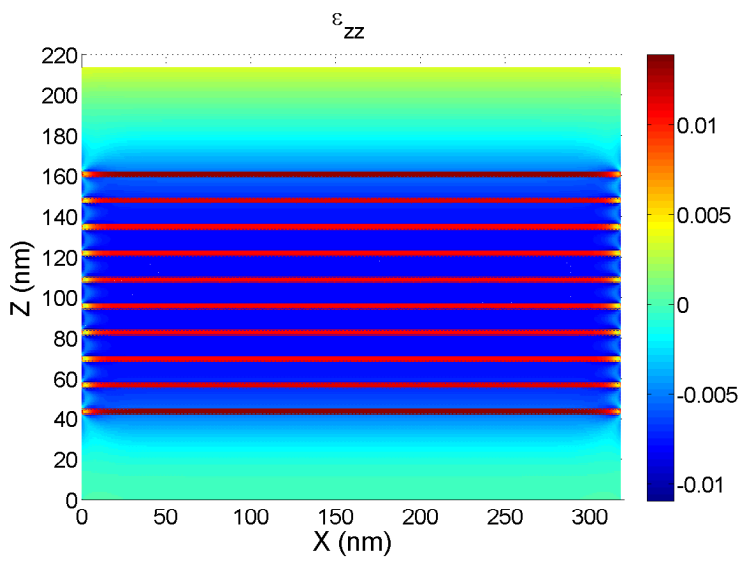

(c)

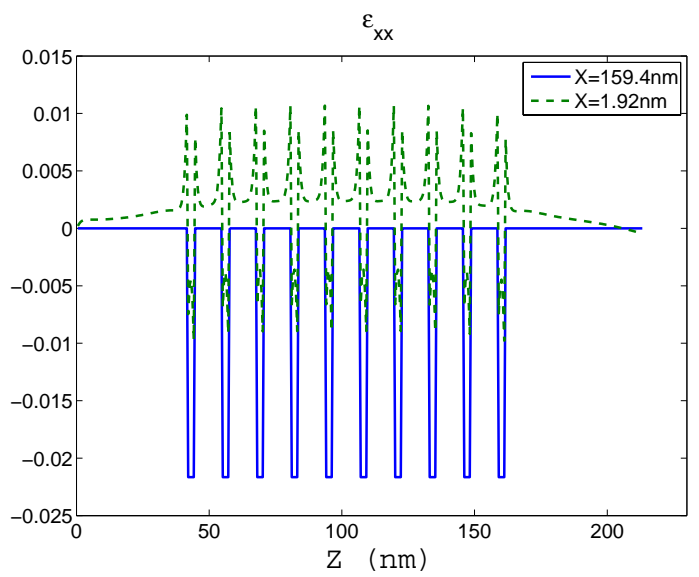

(b)

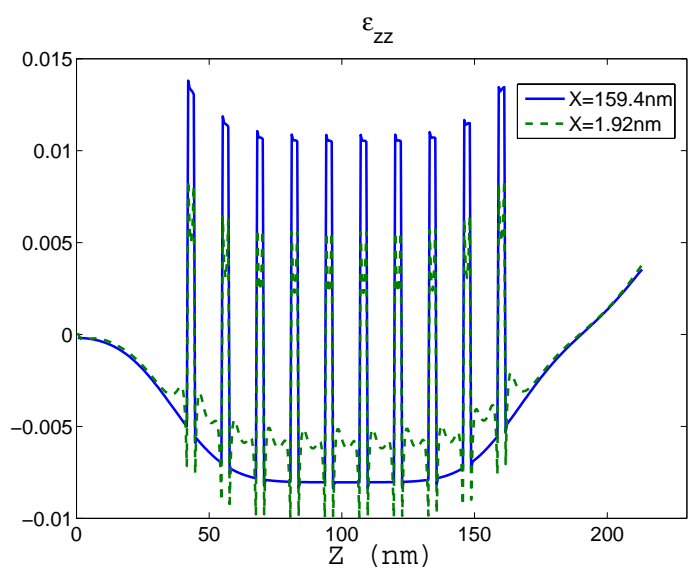

(d)

Figure 3. The color maps show the calculated strain variations in a free-standing, rhombus-shaped $\mathrm{In}_{0.2} \mathrm{Ga}_{0.8} \mathrm{~N} / \mathrm{GaN}$ nanopillar with a height of $Z=210 \mathrm{~nm}$ and an edge width of $X=320 \mathrm{~nm}$ for (a) in-plane strain tensor, $\varepsilon_{x x}$, (b) the $2 \mathrm{~d}$ plot of strain tensor, $\varepsilon_{x x}$, along z-direction at $X=1.92 \mathrm{~nm}$ and $X=159.4 \mathrm{~nm}$ (center), (c) vertical strain tensor, $\varepsilon_{z z}$, and (d) the $2 \mathrm{~d}$ plot of strain tensor, $\varepsilon_{z z}$, along z-direction at $X=1.92 \mathrm{~nm}$ and $X=159.4 \mathrm{~nm}$ (center).

spectrum and the changes of the full width half maximum(FWHM). The emission rate of multiple quantum wells inside the nanopillar can be expressed as

$$
\begin{aligned}
R_{\text {spon }}= & \int d(\hbar \omega) \frac{e^{2} n_{r} \hbar \omega}{m_{0}^{2} \varepsilon_{0} c^{3} \hbar^{2}} \sum_{i, j} \int \frac{2}{(2 \pi)^{2}} d \mathbf{k}\left|\hat{a} \cdot \mathbf{p}_{\mathbf{i}, \mathbf{j}}\right|^{2} \times \\
& \frac{1}{\sqrt{2 \pi} \sigma} \exp \left(\frac{-\left(E_{i, j}-\hbar \omega\right)^{2}}{2 \sigma^{2}}\right) f^{e}\left(E_{i}^{e}(\mathbf{k})\right) f^{h}\left(E_{j}^{h}(\mathbf{k})\right) \quad\left(\mathrm{cm}^{-2} \mathrm{eV}^{-1} \mathrm{~s}^{-1}\right),
\end{aligned}
$$

where $E_{i, j}$ is the average energy separation between electron state $i$ and hole state $j, n_{r}$ is the refractive index, and $\sigma$ is the inhomogeneous broadening factor. $f^{e}$ and $f^{h}$ are the occupancy of electrons and holes. $\left|\hat{a} \cdot \mathbf{p}_{\mathbf{i}, \mathbf{j}}\right|^{2}$ is the momentum matrix element between electronic state, $i$, and hole state, $j$. With the electronic band structures and the energy states obtained from the self-consistent solver, we can apply Eqn. (4) to study the strength of emission spectrum, wave length shift, as well as the screen effects of carrier injection ,and the band filling effects. 


\section{RESULTS AND DISCUSSION}

Figure 2 shows the measured $\mu$-PL spectra. The excitation power is $10 \mathrm{~mW}$. Figure. 2 (a) and (b) are for the device with and without $\mathrm{Pt}$ mask, respectively. The respective CCD images with a scanning area of 5x5 $\mu \mathrm{m}^{2}$ are inserted as the insets. As shown in the inset of Fig. 2(a), the photoluminescence from the freestanding nanopillar is weak, since the Pt mask blocks a significant amount of the excitation on to the nanopillar. Moreover, since the measured spectrum is very similar to that of the as-grown planar structure, we believe that the photoluminescence is mainly contributed by the emission from the periphery of the well. After the mask is removed, the photoluminescence from the nanopillar becomes comparable to that from the well region, as shown in the inset of Fig. 2(b). However, the spectrum without the Pt mask shows additional photoluminescence in the shorter wavelength region, which is likely to originate from the free-standing nanopillar. The measured spectra are then analyzed by the Lorentzian curve fitting. As shown in Fig. 2, two and three dominant Lorentzians are found for the device with and without the mask, respectively, denoted as Lorentzian 1,2 , and 3 . The peak wavelengths and the FWHMs of the Lorentzian 1 and 2 in Fig. 2(a) are nearly identical to those in 2(b), confirming that both of them are photoluminescence from the well region. Therefore, it is concluded that the Lorentzian 3 is the PL originating from the free-standing nanopillar. Since the nanopillar experiences different strain distribution from that of the as-grown planar structure, we hereafter refer to Lorentzian 3 as the PL from the strain-relaxed region of the nanopillar and Lorentzian 1 as that from the strained region. Lorentzian 2 is not discussed here due to its relatively small PL intensity. The peak wavelength and the FWHM of Lorentzian 3 are $435.3 \mathrm{~nm}$ and $30 \mathrm{~nm}$, while those of the Lorentzian 1 are $446 \mathrm{~nm}$ and $22 \mathrm{~nm}$, respectively. The PL from the strain-relaxed region of the nanopillar shows a blue shift of $\sim 68 \mathrm{meV}$ in energy and a FWHM slightly broadened by $\sim 72 \mathrm{meV}$, compared to those from the strained region.

There are several possible reasons for the blue shift and spectra broadening effect. One may be due to the quantum confined effect in the nanopillar. However, the diameter of the nano-pillar is around 300nm, which is too large to induce the quantum confined effect. The other may be due to the strain-related issues. As we know, for the InGaN/GaN quantum well structures grown along c-axis, there exists a strong strain induced piezoelectric polarization field inside the device. This will contribute to the band bending and red shift of spectrum, which is well known as quantum confined Stark effects. The strain in the quantum well might be relaxed at the surface of the nanopillar, which might reduce the polarization field and contribute to the blue shift of the spectrum as well as the emission properties. It is therefore of great interest to investigate the strain variation in the nanopillar. In order to study the strain distribution of the multiple quantum well inside the nanopillar structure, we apply the valence force field model as shown by Eqn. (1). Once the atoms' positions are known, calculating the strain tensor distribution is straightforward with this model. To maintain the symmetry in a wurtzite structure, freestanding, rhombus-shaped GaN pillars consisting of 10-pair $\mathrm{In}_{0.2} \mathrm{Ga}_{0.8} \mathrm{~N}$ MQWs are defined with various edge widths ranging from $60 \mathrm{~nm}$ to $320 \mathrm{~nm}$. The pillars have a fixed boundary condition at the bottom and free boundary conditions on other sides. Figure 3 shows the calculated strain-distribution. Figure 3(a) and 3(c) show the strain tensor, $\varepsilon_{x x}$ and $\varepsilon_{z z}$, of the cross section of x-z plane at the center of y axis, respectively. Figure $3(\mathrm{~b})$ and $3(\mathrm{~d})$ show the line plot at $X=1.92 \mathrm{~nm}$ and $X=159.4 \mathrm{~nm}$ (center) of strain tensor, $\varepsilon_{x x}$ and $\varepsilon_{z z}$, respectively. Both strain tensors show considerable relaxations on the outer shell of the nanopillar, and as-grown-like strain distributions in the center of the nanopillar. The other in-plane strain tensor, $\varepsilon_{y y}$ has a similar distribution to that of $\varepsilon_{x x}$. As shown in Fig. 3(a), the strain relaxation of $\varepsilon_{x x}$ occurs at a distance of $40 \mathrm{~nm}$ from the edge of the nanopillar. At $X=1.92 \mathrm{~nm}$, where the change of strain from the pillar center is most significant, there exist substantial strain variations on the $\mathrm{QW} /$ barrier interfaces and a slight tensile strain of $\varepsilon_{x x}$ in the barriers. We also observe a vertical distribution of $\varepsilon_{z z}$ in the $\mathrm{Z}$ direction, mainly arising from the stacking of MQWs. Both in-plane and vertical strain tensors contribute to the polarization field and can be calculated by Eqn. (2). Based on the calculated strain shown in Fig. 3 and the induced polarization charge calculated by Eqn. (3), we can put this information into the self-consistent Poisson, drift-diffusion, and Schrodinger solver to calculate the band structures of the multiple quantum wells. The parameters we will need for the calculation can be found in Ambacher et. al. ${ }^{15}$

Figure 4 shows the calculated band structure of 10 pair MQW structure. The strain induced polarization charge as a function of depth is fully considered in the simulation. As shown in Fig. 4, the band bending at the center of the nanopillar is the strongest while the band bending at the edge is weakest at $X=1.92 n m$, where

Proc. of SPIE Vol. 7058 70580G-5 

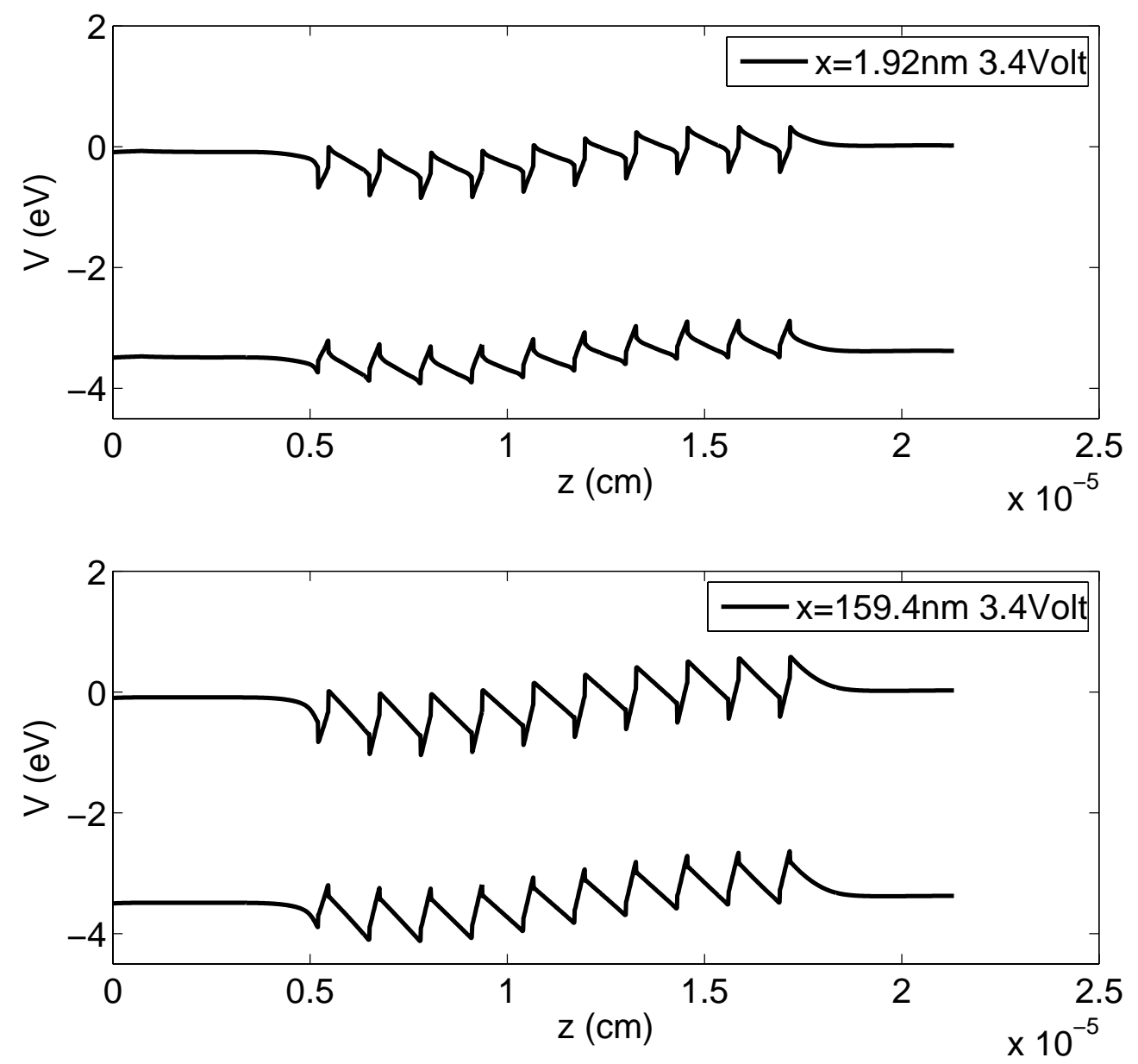

Figure 4. The calculated conduction and valence band profile of the multiple quantum well structures along z-direction at $x=1.92,159.4 \mathrm{~nm}$, respectively.

the strain relaxation reaches the maximum. In order to compare the strength of the emission and compare the energy shift of emission peak at different regions, we use the eigen function and eigen levels obtained from Eqn. (4) to calculate the emission strength and energy shift. The results are shown in Fig. 5.

Figure 5(a) shows the calculated emission spectra of the quantum well at different regions when the total injected electron density at $10^{13} \mathrm{~cm}^{-2}$. As shown in Fig. 5(a), the emission strength near the edge is the strongest. For $X>30 \mathrm{~nm}$, the emission strength does not change too much. And this can be explained from Fig. 3 that strain difference for $X>30 \mathrm{~nm}$ is small compared to the center. The second is to compare the energy shift at different location. Figure 5(b) shows the emission peak shift for the total injected electron density at $10^{13} \mathrm{~cm}^{-2}$. The maximum emission peak shift is occurred at $X=1.92 \mathrm{~nm}$ where there is $189 \mathrm{meV}$ blue shift compared to the center of the nanopillar. At $X=0.32 \mathrm{~nm}$, the strain relaxation is not the maximum at the surface so that the energy shift maximum is not occurred here. Figure 5 (c) shows the emission spectra peak versus different location, $X$. The emission strength reaches the maximum at edge and decrease rapidly from $X=0 \mathrm{~nm}$ to $X=30 \mathrm{~nm}$. The emission strength is almost constant after $X>30 \mathrm{~nm}$, where the electron-hole overlap does not change to much here. Since the center of MQWs still suffers from strain with its magnitude comparable to that of the as-grown planar structure, the photoluminescence from the center of the pillar contributes to the red-edge emission, which is also partially overlapped with the PL from the strained region. Therefore, we believe that 


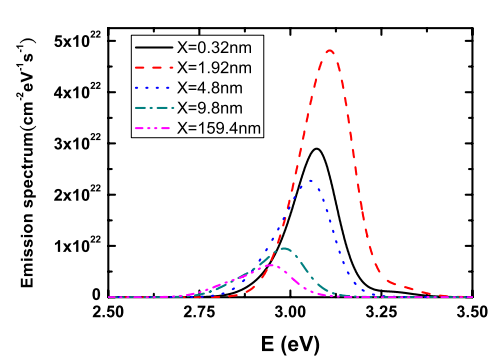

(a)

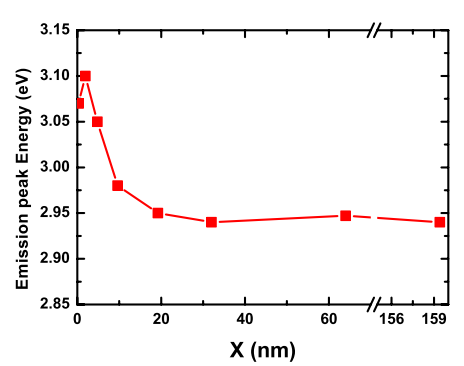

(b)

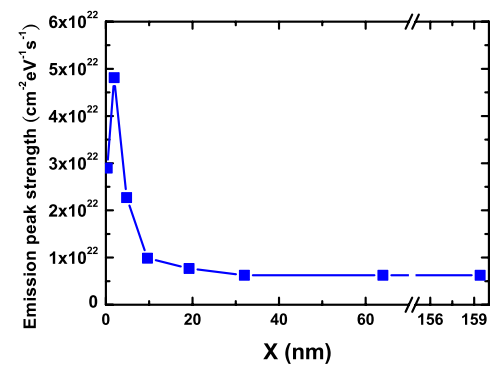

(c)

Figure 5. (a)The calculated spontaneous emission rates as a function of emission energy at different position of nano-pillar with the same injected electron density at $10^{13} \mathrm{~cm}^{-2}$; (b) The calculated spectrum peak shift from the center $X=159.4 \mathrm{~nm}$ to the edge. The maximum strain relaxation region is at $X=1.92 \mathrm{~nm}$, where we obtain the maximum energy shift; and (c) the calculated emission peak strength from the center $X=159.4 \mathrm{~nm}$ to the edge.

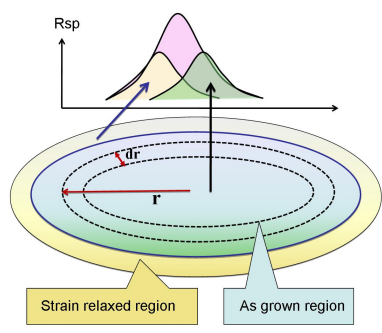

(a)

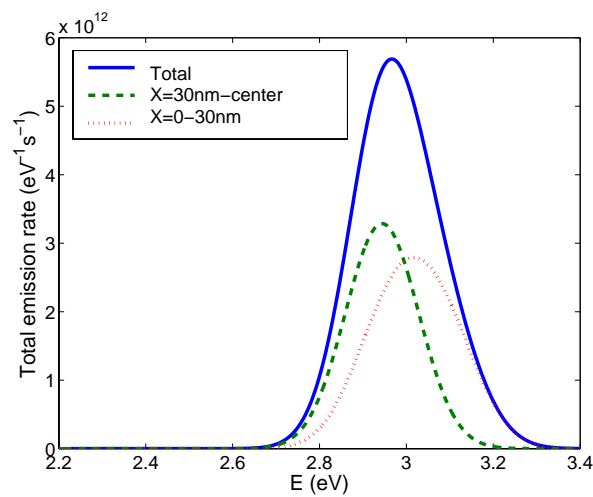

(b)

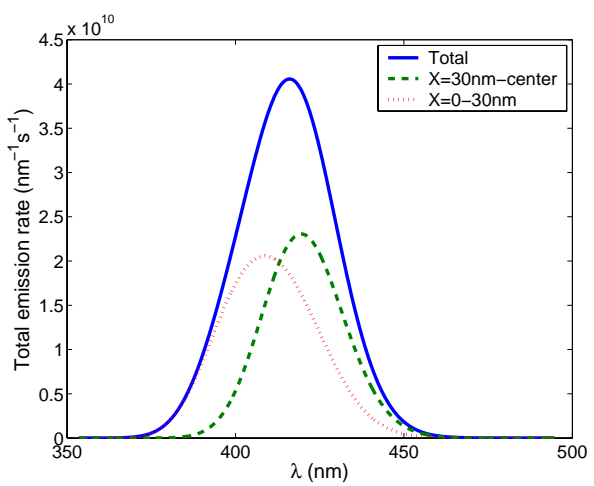

(c)

Figure 6. (a) A schematic of how we estimate the total emission spectrum, (b) The calculated summation of emission spectrum by weighting the emission area size versus energy in $\mathrm{eV}$, and (c) The calculated summation of emission spectrum by weighting the emission area size versus wavelength in $\mathrm{nm}$.

the majority of the nanopillar photoluminescence blue shift originates from the strain-relaxed region, within a distance of $\sim 30 \mathrm{~nm}$ near the edge of the nanopillar as shown in Fig.5 (b). Moreover, the variation of strain tensors in the relaxation region results in a spatially-varied polarization field, leading to the broadened emission spectrum.

The final emission spectrum can be calculated by summation of the emission spectra at different area of each region as shown in Fig 6(a). Therefore, the total emission strength $R_{s p, t o t a l}(\hbar \omega)$ can be obtained by

$$
R_{s p, t o t a l}=\int_{0}^{d} R_{s p}(\hbar \omega, r) 2 \pi r d r \quad\left(\mathrm{eV}^{-1} \mathrm{~s}^{-1}\right)
$$

where $d$ is the radius of the nanopillar and $r$ is the distance to the center of the nanopillar, which is equal to $159.4-X$. The $R_{s p}(\hbar \omega, r)$ can be expressed by a Gaussian approximation where

$$
R_{s p}(\hbar \omega, r)=R_{s p, p e a k}(r) \exp \left(\frac{-\left(\hbar \omega-E_{p e a k}(r)\right)^{2}}{2 \sigma^{2}}\right) \quad\left(\mathrm{cm}^{-2} \mathrm{eV}^{-1} \mathrm{~s}^{-1}\right) .
$$

$R_{s p, p e a k}(r)$ and $E_{\text {peak }}(r)$ are the emission peak strength and the peak energy, respectively, which can be obtained by interpolating the data as shown in Fig. 5(b) and Fig. 5(c). Figure 6(b) and 6(c) show the summation of the emission spectrum in Energy $(\mathrm{eV})$ and in wavelength $\lambda(\mathrm{nm})$ by following Eqn. (5), respectively. The fitting parameter $\sigma$ is set to be $84 \mathrm{meV}$ in order to fit the experimental spectrum width as shown in Fig. 2. The red 
(a)

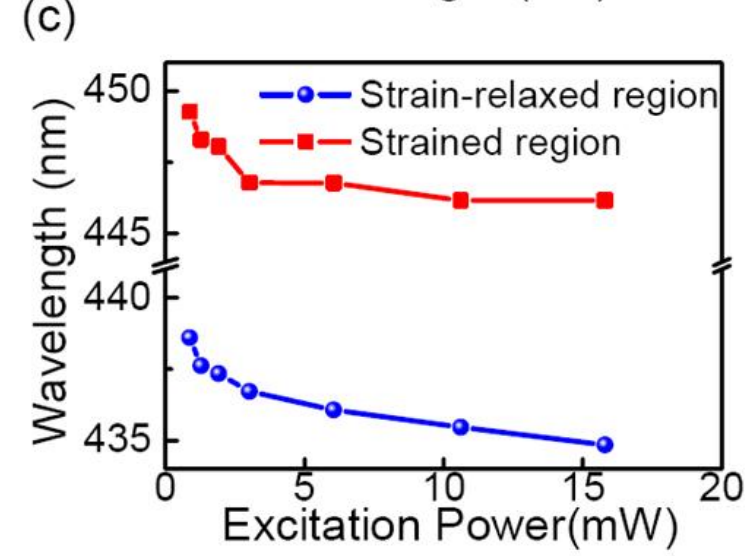

(b)

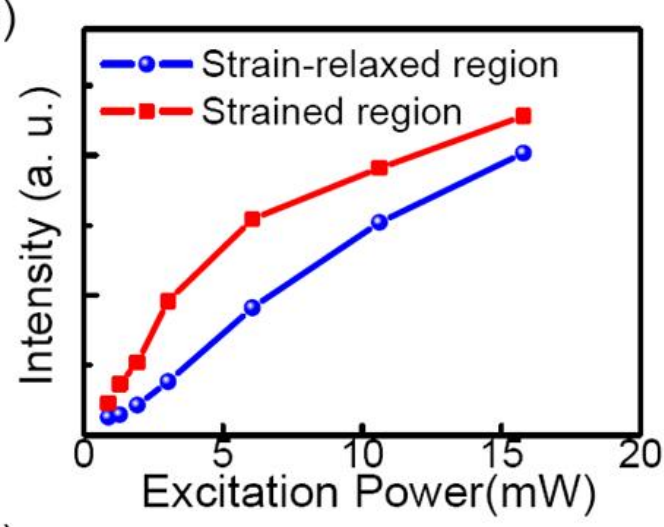

(d)

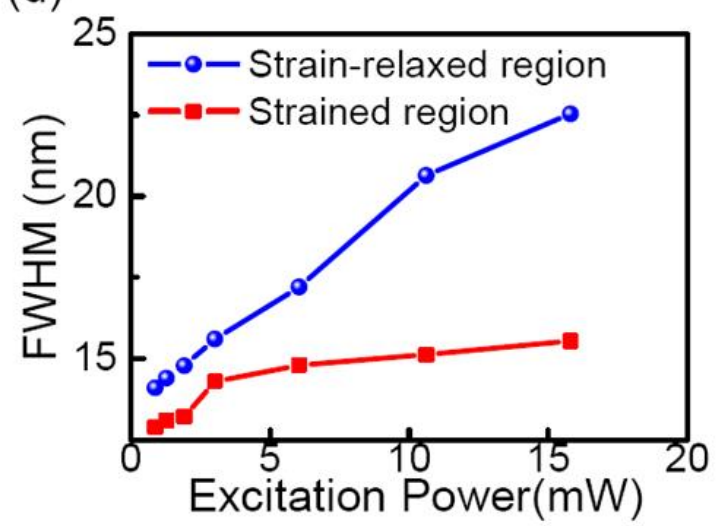

Figure 7. (a) The measured power-dependent $\mu$-PL spectra of the device without the Pt mask with excitation powers ranging from 0.88 to $15.83 \mathrm{~mW}$. Lorentzian analyses of the measured spectra are conducted and plotted for (b) the peak intensity, (c) the emission wavelength, and (d) the FWHM of the PL from the strained and strain-relaxed regions. ${ }^{13}$

dot line is the summation of the emission spectrum from $X=0 \mathrm{~nm}$ to $X=30 \mathrm{~nm}$ and the green dash line is the summation of the emission spectrum from $X=30 \mathrm{~nm}$ to $X=159.4 \mathrm{~nm}$ The two peak difference is around 74 $\mathrm{meV}$, which is close to experimental fitting of $68 \mathrm{meV}$. Therefore, we think that the Lorentz 3 curve as fitted in Fig. 2 is mainly from the emission within the $30 \mathrm{~nm}$ from the edge. Note that if we compare Fig. 2 (b) to Fig.6 (c), there is a 20nm difference of total emission peak from experimental one to the theoretical result. We think this may due to estimated errors of the indium composition, which might be slightly larger than $20 \%$ of indium in the quantum well. It might also be from the estimation error of InGaN bandgap in the simulation, where a linear interpolation is used to calculate the bandgap. However, the overall trend is matched with the experimental result.

Figure $7(\mathrm{a})$ shows the measured power-dependent $\mu$-PL spectra of the device without the Pt mask with excitation powers varying from 0.88 to $15.83 \mathrm{~mW}$. The emission peak shows a slightly blue shift as expected due to the carrier screening of QCSE. The peak intensities, emission wavelengths, and FWHMs of the PL from the strained and the strain-relaxed regions are derived from the Lorentzian fitting of the measured spectra, as plotted in Fig. 7(b) to 7(d) respectively. As seen in Fig. 7(b), the PL intensity of the strained region first increases with excitation power, and then gradually saturates for excitations larger than $6.05 \mathrm{~mW}$. The saturation can also be observed in the wavelength shift and the FWHM, as shown in Fig. 7(c) and 7(d), respectively. On the other hand, the peak intensity and the FWHM of the PL from the strain-relaxed region are monotonically increasing with the excitation. The wavelength blue shift is also more significant than that of the strained region. As the carrier density in InGaN MQWs increases with the incident power, the emission wavelength shifts towards smaller wavelengths and the spectrum becomes broader due to band filling and the screening effect of the piezoelectric field. ${ }^{1}$ However, the radiative recombination process can reduce the band filling effect in MQWs. As shown in 
Fig.5 (a), the estimated emission strength due to the strain relaxation is around 5 times larger than the emission at the center of the nano-pillar. Therefore, to saturate the emission at the strain relaxation region, we will need much higher power (i.e. $>6.05 \mathrm{~mW}$ in this cases). Figure $7(\mathrm{~b})$ to $7(\mathrm{~d})$ suggest that the transition bands are nearly filled in the strained region, where the peak intensity, wavelength shift, and the FWHM vary slowly under high excitation powers. Since the PL from the strain-relaxed region still exhibits the band filling effect under the same excitation condition as the strained region, it is concluded that the radiative recombination rate of the strain-relaxed region is faster than that of the strained region. This is also observed in the simulation results.

Although the strain-relaxed region only occurs at a thin outer shell of the nanopillar - making the direct measurement of the spontaneous emission rate difficult -we believe that fully strain-relaxed nanopillars with a diameter less than $80 \mathrm{~nm}$ have great potential for highly efficient UV/blue light sources. Many studies have also been made on the power-dependent PL characteristics of an ensemble of nanorods with embedded InGaN MQWs,${ }^{1,17}$ where the screening effect, band-filling, and the activation energy of localized indium clusters have been discussed. However, since the power density of a $\mu$-PL measurement is approximately four orders of magnitude larger than that of a conventional PL measurement, only the dominant band-filling effect is taken into account.

\section{CONCLUSION}

In conclusion, we have built a model to analyze the strain distribution and band profile of the MQWs inside the nanopillar structures. Our calculation shows that the energy shift and spectrum broadening could be strongly related to the strain relaxation at nanopillar edge $(\sim 30$ to $40 \mathrm{~nm}$ from the edge). Our calculation also suggests that the emission strength is much stronger at the edge compared to the center, which is also observed in the experimental results. It is also possible that other processes including photon extraction and non-radiative recombination may also be involved and further studies are necessary to completely clarify the emission mechanism. Nonetheless, we believe that strain-relaxed InGaN-based nanopillars can be a promising candidate for highly efficient UV/blue light sources.

\section{ACKNOWLEDGMENT}

The authors would like to thank National Science Council in Taiwan for the financial support, under grant number, NSC96-2218-E-002-031, NSC96-2120-M009-006, and MOEA project under Contract number 95-EC-17A-07-S1-011.

\section{REFERENCES}

[1] Chen, J. H., Feng, Z. C., Tsai, H. L., Yang, J. R., Li, P., Wetzel, C., Detchprohm, T., and Nelson, J., "Optical and structural properties of InGaN/GaN multiple quantum well structure grown by metalorganic chemical vapor deposition," Thin Solid Films 498(1-2), 123-127 (2006).

[2] Yu, C. C., Chu, C. F., Tsai, J. Y., Huang, H. W., Hsueh, T. H., Lin, C. F., and Wang, S. C., "Gallium nitride nanorods fabricated by inductively coupled plasma reactive ion etching," Japanese Journal of Applied Physics Part 2-Letters 41(8B), L910-L912 (2002).

[3] Han, W. Q., Fan, S. S., Li, Q. Q., and Hu, Y. D., "Synthesis of gallium nitride nanorods through a carbon nanotube-confined reaction," Science 277(5330), 1287-1289 (1997).

[4] Kim, H. M., Kim, D. S., Kim, D. Y., Kang, T. W., Cho, Y. H., and Chung, K. S., "Growth and characterization of single-crystal GaN nanorods by hydride vapor phase epitaxy," Applied Physics Letters 81(12), 2193-2195 (2002).

[5] Han, W. Q. and Zettl, A., "Pyrolysis approach to the synthesis of gallium nitride nanorods," Applied Physics Letters 80(2), 303-305 (2002).

[6] Wu, Y. R., Singh, M., and Singh, J., "Device Scaling Physics and Channel Velocities in AlGaN-GaN HFETs: Velocities and Effective Gate Length," IEEE Trans. Electron Devices 53, 588-593 (April 2006).

[7] Palacios, T., Snow, E., Pei, Y., Chakraborty, A., Keller, S., Denbaars, S. P., and Mishra, U. K., "Ge-Spacer Technology in AlGaN/GaN HEMTs for mm-Wave Applications," IEEE IEDM Digest (Dec. May-July 2005). 
[8] Han, S., Jin, W., Zhang, D. H., Tang, T., Li, C., Liu, X. L., Liu, Z. Q., Lei, B., and Zhou, C. W., "Photoconduction studies on GaN nanowire transistors under UV and polarized UV illumination," Chemical Physics Letters 389(1-3), 176-180 (2004).

[9] Johnson, J. C., Choi, H. J., Knutsen, K. P., Schaller, R. D., Yang, P. D., and Saykally, R. J., "Single gallium nitride nanowire lasers," Nature Materials 1(2), 106-110 (2002).

[10] Son, M. S., Im, S. I., Park, Y. S., Park, C. M., Kang, T. W., and Yoo, K. H., "Ultraviolet photodetector based on single GaN nanorod p-n junctions," Materials Science $\& 3$ Engineering C-Biomimetic And Supramolecular Systems 26(5-7), 886-888 (2006).

[11] Kim, H. M., Cho, Y. H., Lee, H., Kim, S. I., Ryu, S. R., Kim, D. Y., Kang, T. W., and Chung, K. S., "Highbrightness light emitting diodes using dislocation-free indium gallium nitride/gallium nitride multiquantumwell nanorod arrays," Nano Letters 4(6), 1059-1062 (2004).

[12] Han, W. Q., Fan, S. S., Li, Q. Q., Gu, B. L., Zhang, X. B., and Yu, D. P., "Synthesis of silicon nitride nanorods using carbon nanotube as a template," Applied Physics Letters 71(16), 2271-2273 (1997).

[13] Yu, P., Chiu, C. H., Wu, Y.-R., Yen, H. H., Chen, J. R., Kao, C. C., Yang, H.-W., Kuo, H. C., Lu, T. C., and Wang, S. C., "Strain relaxation induced micro-photoluminescence characteristics of a single InGaN-based nanopillar fabricated by focused ion beam milling," Appl. Phys. Lett. , Accepted (2008).

[14] Mattila, T. and Zunger, A., "Predicted bond length variation in wurtzite and zinc-blende InGaN and AlGaN alloys," J. Appl. Phys. 85, 160-167 (Jan 1999).

[15] Ambacher, O., Majewski, J., Miskys, C., Link, A., Hermann, M., Eickhoff, M., Stuzmann, M., Bernardini, F., Fiorentini, V., Tilak, V., Schaff, B., and Eastman, L. F., "Pyroelectric properties of Al(In)GaN/GaN hetero- and quantum well structures," J. Phys.: Condens. Matter 14, 3399-3434 (2002).

[16] Wu, Y.-R., Singh, M., and Singh, J., "Gate leakage suppression and contact engineering in nitride heterostructures," J. Appl. Phys. 94, 5826-5831 (November 2003).

[17] Chiu, C. H., Lo, M. H., Lai, C. F., Lu, T. C., Huang, H. W., Chang, Y. A., Hsueh, T. H., Yu, C. C., Kuo, H. C., Wang, S. C., Lin, C. F., and Kuo, Y. K., "Optical properties of $\operatorname{In}_{0.3} \mathrm{Ga}_{0.7} \mathrm{~N} / \mathrm{GaN}$ green emission nanorods fabricated by plasma etching," Nanotechnology 18(33), 335706-335709 (2007). 